Abel, Borel, Cesdro, Hölder and Hausdorff, Journal d'Analyse Mathématique vol. 3 (1953-1954) pp. 346-381.

2. On a converse of Abel's theorem, Proc. Amer. Math. Soc. vol. 3 (1952) pp. 244-256.

3. W. W. Rogosinski, On Hausdorf methods of summability, Proc. Cambridge Philos. Soc. vol. 38 (1942) pp. 166-192.

4. O. Szász, On the product of two summability methods, Annales de la Societé Polonaise de Mathématique vol. 25 (1952-1953) pp. 75-84.

Tel-Aviv, IsRael

\title{
ON A THEOREM OF J. L. WALSH
}

\section{ARYEH DVORETZKY}

1. In a recent paper [1] J. L. Walsh proved, among other results, the following theorem:

Let the functions $f_{n}(x)(n=1,2, \cdots)$ and $f(x)$ be $p$ times differentiable in the interval $a<x<b$ and let $f_{n}(x)$ converge to $f(x)$ in this interval. Then, given any point $x_{0} \in(a, b)$ there exists a sequence of points $x_{n} \in(a, b)$ such that

$$
\lim _{n=\infty} x_{n}=x_{0}, \quad \lim _{n=\infty} f_{n}^{(p)}\left(x_{n}\right)=f^{(p)}\left(x_{0}\right) .
$$

The main purpose of this short note is to show that "in general" there exists a sequence $x_{n}$ satisfying the first condition of (1) and for which $f_{n}^{(p)}\left(x_{n}\right)=f^{(p)}\left(x_{0}\right)$ for all sufficiently large $n$; and when this does not occur then for the corresponding $n$ not only (1) holds but $f_{n}^{(p)}(x)$ is close, in a sense which will be made precise, to $f^{(p)}\left(x_{0}\right)$ in the neighborhood of $x_{0}$. While doing this we shall replace the convergence assumption by a considerably weaker one.

2. Theorem. Let $f(x)$ and $f_{n}(x)(n=1,2, \cdots)$ be $p$ times differentiable in the interval $a<x<b$ and let

$$
\lim _{n=\infty} \operatorname{Inf}_{x \in I, y \in I}\left|f_{n}(y)-f(x)\right|=0
$$

for every open sub-interval I of $(a, b)$. Then, given any point $x_{0} \in(a, b)$, the sequence $N=\{n\}$ can be written as a union of two (not necessarily both infinite) sequences $N_{1}=\left\{n_{1}\right\}$ and $N_{2}=\left\{n_{2}\right\}$ in such a way that

Received by the editors March 28, 1955 and, in revised form, May 23, 1955. 
for every $n_{1}$ there exists $x_{n_{1}} \in(a, b)$ for which

$$
f_{n_{1}}^{(p)}\left(x_{n_{1}}\right)=f^{(p)}\left(x_{0}\right)
$$

and, in case $N_{1}$, is infinite,

$$
\lim _{n_{1}=\infty} x_{n_{1}}=x_{0}
$$

while, if $N_{2}$ is infinite, we have

$$
\limsup _{n_{2}=\infty} \int_{x_{0}-h}^{x_{0}+h}\left|f_{n_{2}}^{(p)}(x)-f^{(p)}\left(x_{0}\right)\right| d x=o(h)
$$

as $0<h \rightarrow 0$. Moreover, if $x_{0}$ is not a local extremum point (in the wide sense) of $f^{(p)}(x)$ then the sequence $N_{2}$ may be taken as finite.

Before proving the theorem we remark that assumption (2) is weaker not only than that of pointwise convergence, but also than those of convergence in measure or convergence on an everywhere dense set. On the other hand the conclusion (5) implies local convergence in measure in the sense that

$$
\lim _{0<h \rightarrow 0} \limsup _{n_{2}=\infty} \mu\left(S_{n_{2}}, \epsilon\right) / 2 h=1
$$

for every $\epsilon>0$, where $\mu\left(S_{n_{2}}, \epsilon\right)$ is the Lebesgue measure of the subset of $\left(x_{0}-h, x_{0}+h\right)$ where $\left|f_{n_{2}}^{(p)}(x)-f^{(p)}\left(x_{0}\right)\right|<\epsilon$.

Proof. We prove the assertion first for $p=1$. We can clearly split $N$ into two disjoint sequences $N_{1}=\left\{n_{1}\right\}$ and $N_{2}=\left\{n_{2}\right\}$ so that $N_{1}$ has the properties stated in the theorem while for every $n_{2}$

$$
f_{n_{2}}^{\prime}(x) \neq f\left(x_{0}\right) \quad \text { for } \alpha<x<\beta,
$$

where $\alpha$ and $\beta$ are fixed points satisfying $a<\alpha<x_{0}<\beta<b$. Our aim is to prove that if $N_{2}$ is infinite then (5) holds. Since derivatives are Darboux functions we have for each $n_{2}$ either $f_{n_{2}}^{\prime}(x)>f^{\prime}\left(x_{0}\right)$ throughout $(\alpha, \beta)$ or $f_{n_{2}}^{\prime}(x)<f\left(x_{0}\right)$ throughout $(\alpha, \beta)$. Splitting $N_{2}$ into two sets $N_{3}=\left\{n_{3}\right\}$ and $N_{4}=\left\{n_{4}\right\}$ according to these two cases, it is necessary to establish the conclusion for each of these sets. For definiteness sake we assume $N_{3}$ infinite and prove

$$
\lim _{n=\infty} \int_{x_{0}-h}^{x_{0}+h}\left|f_{n_{3}}^{\prime}(x)-f^{\prime}\left(x_{0}\right)\right| d x=o(h) .
$$

Given $\epsilon>0$ there exists $h_{0}, 0<h_{0}<(1 / 2) \min \left(x_{0}-\alpha, \beta-x_{0}\right)$ such that 


$$
\left|f\left(x_{0}+t\right)-f\left(x_{0}\right)-t f^{\prime}\left(x_{0}\right)\right|<\epsilon|t|
$$

whenever $|t|<2 h_{0}$. Let $h$ be any number satisfying $0<h \leqq h_{0}$; applying (2) to the intervals $\left(x_{0}-2 h, x_{0}-h\right)$ and $\left(x_{0}+h, x_{0}+2 h\right)$ we see that for all $n>m_{0}=m_{0}(\epsilon, h)$ there exist positive $\bar{\delta}_{n}, \bar{\delta}_{n}^{\prime}, \delta_{n}, \delta_{n}^{\prime}$ all smaller than $\epsilon h /\left(1+\left|f^{\prime}\left(x_{0}\right)\right|\right)$ and for which

$$
\begin{aligned}
& \left|f_{n}\left(x_{0}-h-\bar{\delta}_{n}\right)-f\left(x_{0}-h-\bar{\delta}_{n}^{\prime}\right)\right|<\epsilon h, \\
& \left|f_{n}\left(x_{0}+h+\delta_{n}\right)-f\left(x_{0}+h+\delta_{n}^{\prime}\right)\right|<\epsilon h .
\end{aligned}
$$

Putting $F_{n}(h)=f_{n}\left(x_{0}+h\right)-f\left(x_{0}\right)-h f^{\prime}\left(x_{0}\right)$ and $F(h)=f\left(x_{0}+h\right)-f\left(x_{0}\right)$ $-h f^{\prime}\left(x_{0}\right)$, we obtain from (8) and (9)

$$
\left|F_{n}\left(h+\delta_{n}\right)\right|<\left|F\left(h+\delta_{n}^{\prime}\right)\right|+\left|\delta_{n}-\delta_{n}^{\prime}\right|\left|f^{\prime}\left(x_{0}\right)\right|+\epsilon h<4 \epsilon h
$$

and, similarly, $\left|F_{n}\left(-h-\bar{\delta}_{n}\right)\right|<4 \epsilon h$. But for all $n_{3} \in N_{3}$ we have $F_{n_{3}}^{\prime}(t)=f_{n_{3}}^{\prime}\left(x_{0}+t\right)-f^{\prime}\left(x_{0}\right)>0$ for $|t|<2 h$ and hence

$$
\begin{aligned}
\int_{-h}^{h} \mid f_{n_{3}}^{\prime}\left(x_{0}+t\right) & -f_{n_{3}}^{\prime}\left(x_{0}\right) \mid d t \\
< & \int_{-h-\bar{\delta}_{n}}^{h+\delta_{n}} F_{n_{3}}^{\prime}(t) d t=F_{n_{3}}\left(h+\delta_{n}\right)-F_{n_{8}}\left(-h-\bar{\delta}_{n}\right)<8 \epsilon h .
\end{aligned}
$$

$\epsilon>0$ being arbitrary, this establishes (7).

Now assume that $x_{0}$ is not a local extremum of $f^{\prime}(x)$. Then given any open interval $(\alpha, \beta)$ containing $x_{0}$ there exist in it two points $x^{\prime}$ and $x^{\prime \prime}$ for which $f^{\prime}\left(x^{\prime}\right)>f^{\prime}\left(x_{0}\right)>f^{\prime}\left(x^{\prime \prime}\right)$. According to what has already been proved there exist, for all sufficiently large $n$, points $x_{n}^{\prime}$ and $x_{n}^{\prime \prime}$ in $(\alpha, \beta)$ for which $\left|f_{n}^{\prime}\left(x_{n}^{\prime}\right)-f^{\prime}\left(x^{\prime}\right)\right|<f^{\prime}\left(x^{\prime}\right)-f^{\prime}\left(x_{0}\right)$ and $\mid f_{n}^{\prime}\left(x_{n}^{\prime \prime}\right)$ $-f^{\prime}\left(x^{\prime \prime}\right) \mid<f^{\prime}\left(x_{0}\right)-f^{\prime}\left(x^{\prime \prime}\right)$. In other words there exist $x_{n}^{\prime}, x_{n}^{\prime \prime}$ in $(\alpha, \beta)$ for which $f_{n}^{\prime}\left(x_{n}^{\prime}\right)>f^{\prime}\left(x_{0}\right)>f_{n}^{\prime}\left(x_{n}^{\prime \prime}\right)$; using again the Darboux property, we see that there exists $x_{n} \in(\alpha, \beta)$ for which $f_{n}^{\prime}\left(x_{n}\right)=f^{\prime}\left(x_{0}\right)$. This completes the proof for $p=1$.

Let us now assume the theorem true for $p$ and deduce it for $p+1$. In order to do this we merely have to remark that (5), which implies (6), implies in particular (2) with $f_{n}$ and $f$ replaced by $f_{n}^{(p)}$ and $f^{(p)}$ respectively. Thus applying the theorem for the case of first derivatives to $f_{n}^{(p)}$ and $f^{(p)}$ we obtain the required result. Q.E.D.

3. REMARKs. (1) Hardly any change is needed in the proof in order to establish one-sided analogues of our theorem. Thus we may require that $N_{1}$ satisfy, in addition to (4) and (5), also $x_{n_{1}}>x_{0}$ provided we replace $x_{0}-h$ by $x_{0}$ as the lower limit of integration in (5). This can 
be extended to the end points of the interval. Thus the conclusion remains valid also for $x_{0}=a$ provided $f(x)$ and $f_{n}(x)$ are defined also for $x=a, f(x)$ is $p$-times differentiable to the right there, the functions $f_{n}(x)$ are continuous to the right at $x=a$ (they need not be differentiable there), and $f_{n}(a) \rightarrow f(a)$ as $n \rightarrow \infty$.

(2) The theorem extends easily to functions of several variables.

(3) Condition (2) can be further weakened by assuming it to hold not for the functions $f_{n}(x)$ but for $g_{n}(x)=f_{n}(x)-P_{n}(x)$ where $P_{n}(x)$ are any polynomials of degree smaller than $p$.

(4) Simple examples show that, even assuming uniform convergence of $f_{n}(x)$ to $f(x)$, there does not exist any positive function $\phi(h)$ with $\phi(h) / h \rightarrow 0$ as $h \rightarrow 0$ having the property that (5) holds with $o(h)$ replaced by $o(\phi(h))$. Thus the $o(h)$ in (5) is "best possible." A similar remark applies to (6).

(5) A noninductive proof of the theorem can easily be given, and it actually yields somewhat sharper results for $p>1$. We do not develop these results here since they are special cases of ones applying to more general operators, including among others those of fractional differentiation, which seem to merit a special study.

\section{REFERENCE}

1. J. L. Walsh, On continuity properties of derivatives of sequences of functions, Proc. Amer. Math. Soc. vol. 4 (1953) pp. 69-75.

The Hebrew University of Jerusalem 